\title{
Análisis de aspectos tecnológicos y educativos que influyen en el ingreso económico en México
}

\author{
Analysis of Technological and Educational Aspects that Influence in \\ Economic Income in Mexico
}

\section{Análise dos aspectos tecnológicos e educacionais que influenciam a renda econômica no México}

\author{
Martha Jiménez García \\ Instituto Politécnico Nacional, México \\ majimenez@ipn.mx \\ https://orcid.org/0000-0002-8556-2955 \\ María Vianey Espejel García \\ Instituto Politécnico Nacional, México \\ vianey.espejel@gmail.com \\ https://orcid.org/0000-0001-7307-9379
}

\section{Resumen}

El uso generalizado de las tecnologías de la información y comunicación (TIC) ha ocasionado cambios en todas las actividades del ser humano. Dichas transformaciones se expresan de forma especial en los ámbitos educativo y laboral debido a que se encuentran interrelacionados. Diversos factores influyen en el ingreso económico: el nivel académico, la edad y, precisamente, las habilidades en las TIC, entre otros. El objetivo de la presente investigación fue analizar la influencia de aspectos tecnológicos y educativos en el ingreso económico en México con la finalidad de determinar la importancia de estos y así identificar las habilidades que deben desarrollar los individuos para el ambiente laboral. La investigación es transversal. Los datos se obtuvieron mediante una encuesta semiestructurada y se analizaron a través de una regresión lineal múltiple utilizando el método de mínimos 

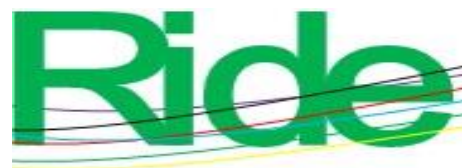

Revista Iberoamericana para la

Investigación y el Desarrollo Educativo

ISSN $2007-7467$

cuadrados ordinarios. En los resultados se encontró que la edad, el uso de redes sociales y el nivel académico $(0.50,0.25,0.24$, respectivamente) son las variables que más influyen positivamente en el ingreso económico de los estudiantes que trabajan. Se concluye que es importante mejorar el nivel educativo y las habilidades en las TIC de la población, pues esto permitirá a los individuos incrementar su ingreso, lo cual coadyuvará a la disminución de la desigualdad.

Palabras clave: edad, impacto, ingreso económico, nivel académico, TIC.

\begin{abstract}
The extensive use of information and communication technologies (ICT) has caused changes in all activities of the human being. Theses changes are expressed in a special way in the educational and labor fields because they are interrelated. Various factors influence economic income such as academic level, age and ICT skills, among others. The objective of this research was to analyze the influence of technological and educational aspects on the economic income in Mexico to determine the importance of these and thus identify the skills that individuals must develop for the work environment. The research is transversal. The data were obtained through a semi-structured survey and were analyzed through a multiple linear regression using the ordinary least squares method. In the results it was found that the age, the use of social networks and the academic level $(0.50,0.25,0.24$, respectively) are the variables that most positively influence the economic income of working students. It is concluded that it is important to improve the educational level and ICT skills of the population, as this will allow individuals to increase their income, which will contribute to the reduction of inequality.
\end{abstract}

Keywords: age, impact, economic income, academic level, ICT.

\title{
Resumo
}


O uso disseminado das tecnologias de informação e comunicação (TIC) tem provocado mudanças em todas as atividades do ser humano. Essas transformações são expressas de maneira especial nos campos educacional e trabalhista porque estão inter-relacionadas. Vários fatores influenciam a renda econômica: o nível acadêmico, a idade e, precisamente, as habilidades em TIC, entre outros. O objetivo desta pesquisa foi analisar a influência de aspectos tecnológicos e educacionais sobre a renda econômica no México, a fim de determinar a importância destes e, assim, identificar as habilidades que os indivíduos devem desenvolver para o ambiente de trabalho. A pesquisa é transversal. Os dados foram obtidos por meio de um levantamento semiestruturado e analisados por meio de uma regressão linear múltipla, utilizando o método dos mínimos quadrados ordinários. Nos resultados verificouse que a idade, o uso das redes sociais e o nível acadêmico $(0,50 ; 0,25 ; 0,24$, respectivamente) são as variáveis que mais influenciam positivamente a renda econômica dos estudantes trabalhadores. Conclui-se que é importante melhorar o nível educacional e as habilidades de TIC da população, pois isso permitirá que os indivíduos aumentem sua renda, o que contribuirá para a redução da desigualdade.

Palavras-chave: idade, impacto, renda econômica, nível acadêmico, TIC.

Fecha Recepción: Febrero 2019

Fecha Aceptación: Junio 2019

\section{Introducción}

Las tecnologías de la información y comunicación (TIC) son aquellas que hacen posible la transmisión, procesamiento y almacenamiento de información en cualquier momento y en cualquier lugar, incluyendo los instrumentos electrónicos que permitan dichos procedimientos (Cacheiro, 2018). El uso generalizado de dichas tecnologías ha ocasionado cambios en todas las actividades del ser humano, los cuales se expresan de forma especial en los ámbitos educativo y laboral.

Ambas esferas, la educativa y la laboral, están interrelacionadas: las capacidades y habilidades desarrolladas mediante la educación deberán corresponder a las requeridas por las instituciones y empresas del ámbito laboral. Por lo tanto, la educación formal complementada con la formación respecto a las TIC permitirá a los individuos incrementar su ingreso (Dutton y Reisdorf, 2016). 


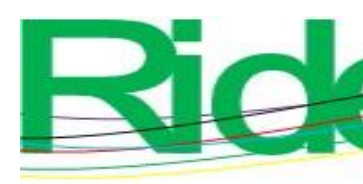

Revista Iberoamericana para la

Investigación y el Desarrollo Educativo

ISSN $2007-7467$

Existen diversos factores que influyen en el ingreso económico de la población mexicana, tales como el nivel educativo, la edad, la experiencia y las habilidades en las TIC, entre otros. Shahabadi, Nemati y Hosseinidoust (2016) indican que para lograr el incremento en el ingreso y lograr disminuir la desigualdad se deberá invertir en capital humano; si se considera el incremento en la adopción de tecnología en diferentes ámbitos económicos, la inversión en capital humano deberá enfocarse en el desarrollo de habilidades relacionadas a las TIC. Considerar dichos factores permitirá identificar ejes temáticos para el desarrollo de políticas que estén encaminadas al incremento de habilidades para lograr disminuir las desigualdades.

Con la finalidad de lograr que un mayor número de personas esté calificado para trabajos con mejores sueldos, fue desarrollado el proyecto Mexico FIRST, el cual ofrece programas de certificación que aumentan la calidad de los recursos humanos mexicanos (Banco Mundial, 2014). Sin embargo, a pesar de dichos esfuerzos, se observa que en 2016 los mexicanos invirtieron poco en educación, pues destinaron solo $12.42 \%$ a servicios educativos y esparcimiento (Instituto Nacional de Estadística y Geografía [Inegi], 2016).

Debido a la importancia del desarrollo de la capacidad de maniobra de las TIC en el ámbito educativo para obtener los beneficios en el mercado laboral y, por tanto, en el ingreso económico, se decidió analizar la influencia de aspectos tecnológicos y educativos en el ingreso económico en México con la finalidad de determinar la importancia de estos y así identificar las habilidades que deben desarrollar los individuos para el ambiente laboral.

Las preguntas de investigación fueron las siguientes: ¿Cómo influyen los aspectos tecnológicos en el ingreso económico?, ¿qué porcentaje de estudiantes universitarios que trabajan utiliza las redes sociales?, ¿qué efecto tiene el uso de las redes sociales en el ingreso económico?, ¿en cuánto afecta la posesión de una Smart TV al ingreso económico?, ¿en cuánto afecta la posesión de un teléfono móvil en el ingreso económico?, ¿cuál es el efecto del uso laboral de dispositivos móviles en el ingreso económico?, ¿cómo influyen los aspectos educativos en el ingreso económico?, ¿cuál es el rango de edad de los estudiantes universitarios que trabajan?, ¿cuál es el efecto de la edad de los estudiantes universitarios que trabajan en el ingreso económico?, ¿cuál es el efecto del nivel académico en el ingreso económico?, ¿cuál es el efecto que tiene el uso escolar de dispositivos móviles en el ingreso económico? 


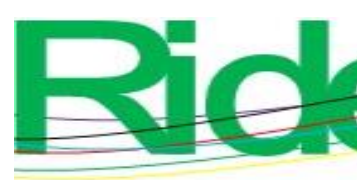

Revista Iberoamericana para la

Investigación y el Desarrollo Educativo

ISSN $2007-7467$

La hipótesis del trabajo es que el nivel académico, la edad y el desarrollo de habilidades en las TIC son importantes para que los individuos puedan incrementar sus ingresos y poder disminuir la desigualdad.

\section{TIC}

Las TIC son consideradas como un conjunto de herramientas transformadoras que puede provocar cambios sistémicos, debido a que conectan a las personas con los empleos, mercados y servicios sociales (Organización de las Naciones Unidas para la Educación, la Ciencia y la Cultura [Unesco], 2015). Por lo anterior, el Banco Mundial (2014) busca respaldar las TIC y el desarrollo de habilidades para los empleos relacionados con estas con la intención de mejorar la productividad, la competitividad y acelerar la innovación en la economía en general.

Las TIC se han propagado en la vida de los mexicanos, ya que en 2001 solo $11.8 \%$ de los hogares contaba con equipo de cómputo, mientras que en el 2017 fueron $45.4 \%$; en el caso del acceso a Internet, el incremento es mayor, pues en 2001 solo $6.2 \%$ de los hogares contaba con este servicio, en cambio, en 2017 dicha cifra se incrementó a $50.9 \%$ (Inegi, 2017).

\section{Educación y TIC}

Las TIC han sido reconocidas como facilitadoras del aprendizaje futuro pues permiten que las personas aprendan en cualquier momento, en cualquier lugar y con todo el contenido que puedan necesitar (Unesco, 2015). Estas herramientas aportan a la educación y a la sociedad flexibilidad, así como la capacidad para adaptarse a un entorno cambiante (Hernández, 2017).

Actualmente, en la agenda política pública de México y de América Latina se consideran las políticas de integración de las TIC en los sistemas educativos, con la finalidad de que los estudiantes tengan la oportunidad de desarrollar habilidades que les permitan mejorar no solo en el ámbito académico, también en el laboral. En 2017, el Inegi reportó que del total de usuarios de computadoras, $46.8 \%$ las utilizaron como herramienta de apoyo escolar. 


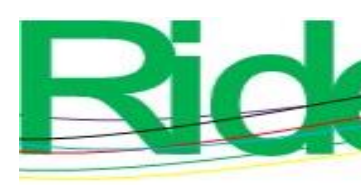

Revista Iberoamericana para la

Investigación y el Desarrollo Educativo

ISSN $2007-7467$

Para manejar adecuadamente las herramientas de información conocidas como TIC se requiere de trabajadores mejor calificados, con niveles de educación más altos y que hayan desarrollado destrezas tecnológicas; evidentemente, el requerir trabajadores mejor calificados, implicará un incremento en los salarios (Mendoça y Crespo, 2015).

Algunos estudios realizados por Dutton y Reisdorf (2016), Shahabadi et al. (2016) y Serrano, Muñoz y Brusca (2018) indican que cuanto mayor es el nivel de estudio del individuo mayor es el nivel de uso del Internet, pues realiza actividades como la recuperación de información, comunicación y acceso a servicios financieros. Lo anterior muestra la importancia del acceso y el desarrollo de habilidades entorno a las TIC, lo cual permitirá especificar el tipo de trabajo y nivel de pago como señal de capacidad y productividad, y lograr así disminuir la desigualdad en los ingresos de la población.

El trabajo y el aprendizaje pueden integrarse a través de las TIC, debido a que estas pueden utilizarse para desarrollar un pensamiento crítico, así como otras capacidades necesarias para trabajar en diversos entornos. Sin embargo, se debe poner especial atención en la manera en la que se enseñan dichas capacidades, pues métodos educativos inapropiados podrían ocasionar un problema significativo de equidad (Unesco, 2015).

\section{Empleo y TIC}

El uso de las TIC influye directamente en el empleo, así como en la inclusión de grupos desfavorecidos en el mercado laboral (Evangelista, Guerrieri y Meliciani, 2014), por lo que se considera que estas pueden ser utilizadas para incrementar el ingreso de los individuos. Sin embargo, Frey y Osborne (2013) indican que las computadoras, como ejemplo de tecnología, constituyen un complemento para los trabajadores altamente calificados y un sustituto para trabajadores que están poco calificados.

Islam y Slack (2016) mencionan que las TIC proporcionan acceso a información que afecta positivamente los medios de vida de la población, principalmente las oportunidades económicas. Las TIC no solo proporcionan información de posibles empleos, también permiten el acceso a información financiera y comercial para emprendedores y autoempleadores. 
En cuanto al uso de las TIC, específicamente en el caso del Internet, este permite al individuo vender su trabajo en línea, obtener información sobre oportunidades de empleo en diferentes industrias y complementar sus ingresos mensuales principalmente a través de la venta de productos (Islam y Slack, 2016; Huws, Spencer y Syrdal, 2018).

\section{Materiales y métodos}

La investigación fue de tipo exploratoria para conocer el impacto de aspectos tecnológicos y educativos que influyen en el ingreso económico; fue un estudio transversal realizado en Ciudad de México en el año 2017. El estudio fue dirigido exclusivamente a estudiantes universitarios que trabajan, incluyendo las siguientes variables independientes: "Smart TV", "Redes sociales", "DM-Uso escolar", "DM-Uso laboral”, "Nivel académico", “Teléfono móvil” y "Edad”. Estas mismas variables se presentan en la figura 1 y se describen en la tabla 1; como variable dependiente se tiene el ingreso económico. Se realizó un muestreo aleatorio simple mediante el cual se obtuvo una muestra de 247 personas que estudian a nivel licenciatura y trabajan en la capital mexicana en el año 2017. En las siguientes secciones se describe el instrumento y el modelo propuesto.

Figura 1. Variables del modelo

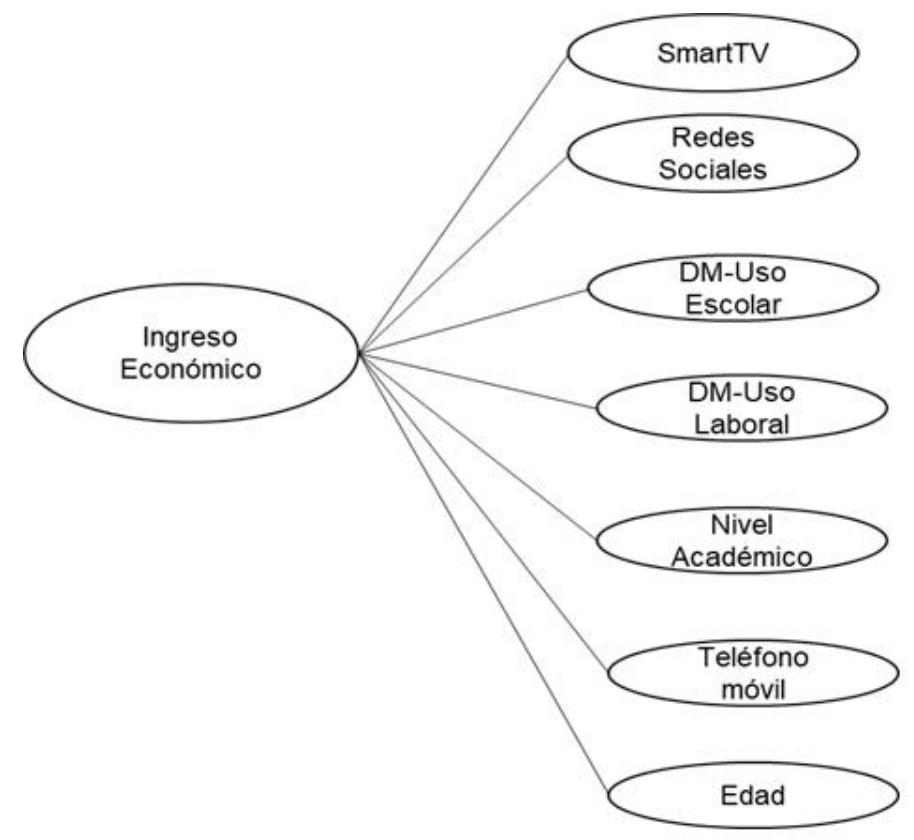

Fuente: Elaboración propia 


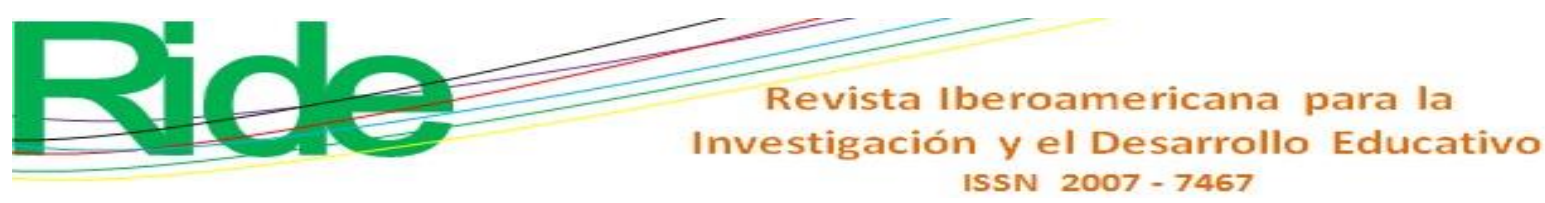

Tabla 1. Explicación de las variables del modelo

\begin{tabular}{|c|c|c|}
\hline Variable & Descripción & Tipo y relación \\
\hline $\begin{array}{l}\text { Ingreso } \\
\text { económico (Y) }\end{array}$ & $\begin{array}{l}\text { Ingreso económico } \\
\text { mensual de personas } \\
\text { que estudian y trabajan. } \\
\text { Esta variable fue } \\
\text { clasificada en ocho } \\
\text { niveles de ingreso } \\
\text { mensual personal }\end{array}$ & $\begin{array}{l}\text { Dependiente: Variable principal para analizar los } \\
\text { factores tecnológicos y de dispositivos móviles (DM) } \\
\text { que influyen en el ingreso económico }\end{array}$ \\
\hline Smart TV & $\begin{array}{l}\text { Personas que tienen una } \\
\text { Smart TV (Televisión } \\
\text { con internet) }\end{array}$ & $\begin{array}{l}\text { Independiente: Variable considerada por ser un } \\
\text { dispositivo que se conecta a Internet y puede influir } \\
\text { en el ingreso económico, pues el tener este tipo de } \\
\text { conexión le puede otorgar al usuario, a través de una } \\
\text { capacitación, mayores habilidades para la vida y el } \\
\text { trabajo }\end{array}$ \\
\hline Redes sociales & $\begin{array}{l}\text { Personas que hacen uso } \\
\text { de las redes sociales }\end{array}$ & $\begin{array}{l}\text { Independiente: Variable considerada debido a que en } \\
\text { las redes sociales se oferta contenido relacionado con } \\
\text { ofertas laborales o capacitación para el trabajo o } \\
\text { estudios y esto puede influir en el ingreso económico }\end{array}$ \\
\hline $\begin{array}{l}\text { DM-Uso } \\
\text { escolar }\end{array}$ & $\begin{array}{l}\text { Dispositivo móvil para } \\
\text { uso escolar }\end{array}$ & $\begin{array}{l}\text { Independiente: Variable tecnológica debido a que se } \\
\text { conecta al Internet y puede buscar contenidos } \\
\text { académicos para actualizar sus estudios, además de } \\
\text { que puede repasar sus notas de estudio y esto influye } \\
\text { en el ingreso económico }\end{array}$ \\
\hline $\begin{array}{l}\text { DM-Uso } \\
\text { laboral }\end{array}$ & $\begin{array}{l}\text { Dispositivo móvil para } \\
\text { uso laboral }\end{array}$ & $\begin{array}{l}\text { Independiente: Variable tecnológica debido a que se } \\
\text { conecta al Internet y puede usarse para actividades } \\
\text { laborales y esto influye en el ingreso económico }\end{array}$ \\
\hline $\begin{array}{l}\text { Nivel } \\
\text { académico }\end{array}$ & $\begin{array}{l}\text { Nivel académico, } \\
\text { incluye primaria, } \\
\text { secundaria, bachillerato } \\
\text { superior y posgrado }\end{array}$ & $\begin{array}{l}\text { Independiente: variable utilizada para ver la relación } \\
\text { entre el nivel académico y el ingreso económico }\end{array}$ \\
\hline Teléfono móvil & $\begin{array}{l}\text { Cantidad de teléfonos } \\
\text { móvil que tiene una } \\
\text { persona }\end{array}$ & $\begin{array}{l}\text { Independiente: variable importante de igual forma } \\
\text { por ser un dispositivo móvil con acceso a internet y a } \\
\text { diversas aplicaciones como las redes sociales, foros, } \\
\text { entre otras }\end{array}$ \\
\hline Edad & $\begin{array}{l}\text { Edad de las personas en } \\
\text { número entero }\end{array}$ & $\begin{array}{l}\text { Independiente: se consideró esta variable para ver la } \\
\text { relación entre ingreso económico y edad }\end{array}$ \\
\hline
\end{tabular}

Fuente: Elaboración propia

\section{Instrumento}

Se utilizó una encuesta semiestructurada como instrumento, con secciones de preguntas que tienen relación con las variables independientes y la variable dependiente. Se aplicó la encuesta de forma aleatoria al tamaño de muestra. El cuestionario cuenta con dos secciones. La primera con preguntas abiertas diseñada para obtener datos generales del individuo: nombre, edad, lugar de procedencia, nivel de estudios, ocupación, etc.; la segunda, 

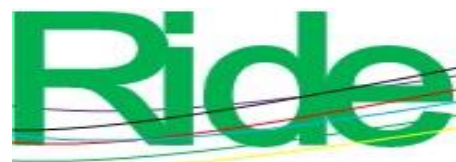

Revista Iberoamericana para la

Investigación y el Desarrollo Educativo

ISSN $2007-7467$

con preguntas cerradas de opción múltiple, captó aspectos económicos del individuo y sobre su manejo de las TIC: ingreso económico, disponibilidad y frecuencia de uso de dispositivos móviles y finalidad del uso de estos. El cuestionario fue validado aplicándolo a una muestra piloto y posteriormente obteniendo el alfa de Cronbach. Se pidió a los encuestados que indicaran la frecuencia de uso de las herramientas tecnológicas (Smart TV, redes sociales) para actividades académicas; asimismo, la encuesta incluye el uso de dispositivos móviles (DM) para uso escolar y laboral, así como datos generales sobre su nivel académico, cantidad de teléfonos móviles y edad. La frecuencia de uso incluyó los números $0,1,2,3,5,6$ y 7, los cuales indican la frecuencia de empleo en días a la semana, en donde 0 es nunca y 1 es un día y así sucesivamente.

Para determinar el impacto que tienen las variables independientes mencionadas anteriormente en el ingreso económico (variable dependiente), se utilizó el concepto de elasticidad. La elasticidad cuantifica el cambio en la variable dependiente ante cambios en las variables independientes, es decir, qué tan sensible es el ingreso económico a Smart TV, redes sociales, entre otros.

La elasticidad (E) en cualquier punto de la curva está dado por (Gujarati, 2010):

$$
E=\left({ }^{\partial Y_{t}} / \partial X_{t-1}\right)\left(X_{t-1} / Y_{t}\right)=b_{1}\left(X_{t-1} / Y_{t}\right)
$$

Donde: $\left(\partial Y_{t} / \partial X_{t}\right)$ es la pendiente de la curva $\left(b_{1}\right)$ y $X_{t}$ y $Q_{t}$ son las medias de las variables independientes y la variable dependiente, respectivamente.

\section{Resultados}

Con las variables identificadas para captar los aspectos tecnológicos y educativos y el ingreso económico, se elaboró un modelo para analizar la interacción de variables: se generó un modelo de regresión lineal múltiple estimado mediante el método de mínimos ordinarios, con el procedimiento correspondiente (MCO) en el software SAS versión 9.0. El análisis realizado se basó en el uso de un estadístico denominado valor-p, el cual corresponde a la probabilidad de aceptar la hipótesis nula, comparada con el nivel de significancia $\alpha$ (se utilizó $\alpha=0.05$ ). Asimismo, la validez del modelo se comprueba con la prueba de $F$. 


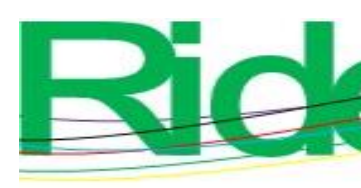

Revista Iberoamericana para la

Investigación y el Desarrollo Educativo

ISSN $2007-7467$

El modelo propuesto de regresión lineal múltiple tiene como variable dependiente el ingreso económico de los estudiantes. Asimismo, se tienen las variables independientes ya especificadas arriba: "Smart TV”, "Redes sociales", "Dm-Uso escolar", "DM-Uso laboral”, "Nivel académico", "Teléfono móvil" y "Edad". El trabajo es especificado bajo la función que se indica en la ecuación 1.

Ingreso Económico $=f($ SmartTV, Redes Sociales, $D M-$ Uso Escolar,DM - Uso Laboral, Nivel Académico,Telefóno móvil, Edad)

También se tiene la ecuación 2, la cual representa el modelo teórico que se plantea y que analiza el ingreso económico con las variables ya mencionadas en la tabla 1.

$$
\begin{gathered}
Y=\beta_{0}+\beta_{1} \text { SmartTV }+\beta_{1} \text { RedesSociales }+\beta_{2} D M-\text { UsoEscolar }+\beta_{3} D M \\
- \text { UsoLaboral }+\beta_{4} \text { Nivel Academico }+\beta_{5} \text { Telefóno móvil }+\beta_{6} \text { Edad } \\
+u_{1} \quad \text { (2) }
\end{gathered}
$$

Se realizó un análisis de fiabilidad de los datos obtenidos del cuestionario considerando el alfa de Cronbach: fue equivalente a 0.811. Del análisis de datos se encontró que los encuestados fueron estudiantes universitarios que trabajan y que tienen entre 18 y 61 años, de los cuales $55 \%$ son hombres. En cuanto al ingreso económico mensual, se observa que la población estudiantil universitaria-trabajadora obtiene en promedio entre 3000 y 7000 pesos. En cuanto al uso de las redes sociales, $69 \%$ de los encuestados refirieron utilizarlas 7 días a la semana, principalmente con fines de comunicación social-laboral y entretenimiento.

El modelo estimado muestra una $F$ calculada de 23 con un valor-p equivalente a 0.000, por lo que se rechaza la hipótesis nula en favor de la hipótesis alternativa y se concluye que al menos uno de los parámetros de la ecuación es diferente de cero.

Los valores de la t de Student indican que todos los coeficientes de las variables explicativas del modelo son estadísticamente significativos, debido a que resultaron mayores a 1 en términos absolutos y con valor-p menor al 0.05; además, los signos concuerdan con el análisis empírico planteado. Por lo tanto, se consideran todas las variables explicativas dentro del modelo. En la tabla 2 se presentan los coeficientes estimados en el modelo. 


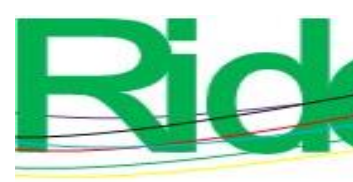

Revista Iberoamericana para la

Investigación y el Desarrollo Educativo

ISSN $2007-7467$

Tabla 2. Resultados estadísticos y coeficientes del modelo en su forma estructural

\begin{tabular}{|c|c|c|c|c|c|c|c|c|}
\hline Variable & Constante & Variab & les indep & endient & & & & \\
\hline $\begin{array}{l}\text { Ingreso } \\
\text { económico }\end{array}$ & & $\begin{array}{l}\text { Smart } \\
\text { TV }\end{array}$ & $\begin{array}{l}\text { Redes } \\
\text { sociales }\end{array}$ & $\begin{array}{l}\text { DM- } \\
\text { Uso } \\
\text { escolar }\end{array}$ & $\begin{array}{l}\text { DM- } \\
\text { Uso } \\
\text { laboral }\end{array}$ & $\begin{array}{l}\text { Nivel } \\
\text { académico }\end{array}$ & $\begin{array}{l}\text { Teléfono } \\
\text { móvil }\end{array}$ & Edad \\
\hline $\begin{array}{l}\text { Coeficiente } \\
\text { estimado }\end{array}$ & 0.029 & 0.366 & 0.142 & -0.816 & 0.833 & 0.481 & -0.121 & 0.067 \\
\hline$T$ & 0.050 & 4.253 & 2.260 & -3.307 & 4.637 & 3.661 & -2.530 & 4.760 \\
\hline Valor-p & 0.960 & 0.000 & 0.025 & 0.001 & 0.000 & 0.000 & 0.012 & 0.000 \\
\hline$F$ & 23.005 & prob $F$ & 0.000 & & & & & \\
\hline
\end{tabular}

Fuente: Elaboración propia con datos del modelo

La estimación de la regresión permite indicar el efecto que tiene el uso de las redes sociales, los dispositivos digitales más utilizados, así como la finalidad de su uso, el nivel académico y la edad en el ingreso mensual personal de los individuos que estudian en el nivel licenciatura y trabajan.

Los coeficientes obtenidos muestran que existe una relación directa entre el ingreso mensual personal y la frecuencia de los días que usan las redes sociales, la cantidad de Smart TV que tienen en su hogar, el uso laboral de los dispositivos digitales, el nivel académico y la edad; en cambio, se observa una relación inversa entre el uso escolar de los dispositivos móviles y la cantidad de teléfonos móviles con la que cuentan los trabajadores que estudian.

En la tabla 3 se muestran las elasticidades que se calcularon tomando en cuenta los parámetros estimados y el promedio de las variables involucradas; lo anterior con la intención de analizar el impacto que tienen las variables en el ingreso mensual personal.

Tabla 3. Elasticidades calculadas

\begin{tabular}{|l|l|l|l|l|l|l|l|}
\hline Variable & $\begin{array}{l}\text { Smart } \\
\text { TV }\end{array}$ & $\begin{array}{l}\text { Redes } \\
\text { sociales }\end{array}$ & $\begin{array}{l}\text { DM-Uso } \\
\text { escolar }\end{array}$ & $\begin{array}{l}\text { DM-Uso } \\
\text { laboral }\end{array}$ & $\begin{array}{l}\text { Nivel } \\
\text { académico }\end{array}$ & $\begin{array}{l}\text { Teléfono } \\
\text { móvil }\end{array}$ & Edad \\
\hline Elasticidad & 0.10 & 0.28 & -0.21 & 0.15 & 0.24 & -0.06 & 0.50 \\
\hline
\end{tabular}

Fuente: Elaboración propia con datos del modelo. 


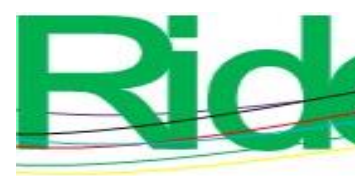

Revista Iberoamericana para la

Investigación y el Desarrollo Educativo

ISSN $2007-7467$

Los resultados obtenidos indican que los dispositivos digitales como la Smart TV tienen un efecto positivo en el ingreso mensual personal. Si la cantidad de Smart TV en el hogar aumentara en $10 \%$, el ingreso mensual personal aumentará en $1 \%$. En el caso del teléfono móvil, este tiene un efecto negativo, ya que un incremento de $10 \%$ en la cantidad de teléfonos móviles ocasionaría una disminución de $0.6 \%$ en el ingreso mensual personal.

\section{Discusión}

En los resultados se encontró que el uso de los dispositivos digitales como la Smart TV contribuye de forma positiva al ingreso económico. En cambio, el teléfono móvil tiene un efecto negativo. Lo anterior puede ser debido a que al implementar medios informáticos los salarios disminuyen y afectan principalmente a los trabajadores que están moderadamente calificados, con lo que se incrementa la desigualdad en el ingreso (Yeaple, 2005; Frey y Osborne, 2013; Santos, Sequeira y Ferreira, 2017). El efecto negativo de la propiedad de teléfonos móviles difiere de lo obtenido, pues diversos autores mencionan que los teléfonos móviles pueden utilizarse para incrementar el ingreso de la población debido a que proveen información sobre oportunidades económicas (Hübler y Hartje 2016; Islam y Slack, 2016). Cabe mencionar que para obtener los beneficios de las TIC se deberá de desarrollar habilidades para su manejo. Y actualmente la mejora en dispositivos móviles ha ocasionado que los individuos tengan que mejorar sus habilidades constantemente.

Se observa también que los días de uso de redes sociales influyen positivamente en el ingreso personal mensual; si los días de uso incrementan en $10 \%$, entonces el ingreso personal mensual aumentará en $2.8 \%$. Considerando que el uso de redes sociales es una de las variantes de la riqueza informativa, los datos concuerdan con la Comisión Europea, quien menciona que el ingreso per cápita se correlaciona positivamente con la riqueza informativa (Mendoça y Crespo, 2015). Sin embargo, Wu (2013) indica que en su estudio no se encontró correlación entre los ingresos facturables futuros y el uso de una red social dentro de una organización.

En el caso del uso de dispositivos tecnológicos, se observa que el uso escolar de dichos dispositivos influye negativamente en el ingreso mensual personal; en cambio, el uso laboral de los dispositivos digitales influye de manera positiva. Si el uso escolar aumenta en $10 \%$, entonces el ingreso personal mensual disminuirá en $2.1 \%$. Lo anterior concuerda con 
lo mostrado en el estudio de Álvarez, Vega y Álvarez (2011), quienes indican que la formación que los actores reciben en las universidades e instituciones de enseñanza no es valorada como algo útil ya que el afianzamiento en el manejo de las TIC se dio fuera de la escuela. Por su parte, la Unesco (2015) indica que la forma en la que se enseñan las habilidades en las TIC puede ocasionar un problema de equidad significativo al estar ligadas estas con el nivel socioeconómico de los individuos. Por lo tanto, se deberá poner atención en la forma en la que se está disminuyendo la brecha digital, no solo en cuestiones de acceso al hardware y al desarrollo de habilidades para poder explotar el uso de las TIC.

Y por el contrario, si el uso laboral es el que aumenta, entonces el ingreso personal mensual aumentará en $1.5 \%$. Lo anterior muestra la importancia de los dispositivos digitales como herramienta para incrementar el ingreso de diferentes sectores y la manera en que las TIC están penetrando en todos los aspectos de la vida cotidiana. Esto concuerda con lo mencionado por Huws et al. (2018), quienes indican que en Europa algunos trabajadores obtienen ingresos complementarios al vender su fuerza de trabajo mediante el uso de dispositivos móviles, así como redes sociales. Por su parte, Álvarez et al. (2011) indican que algunos emprendedores consideran a las TIC como un factor importante para que crezcan sus negocios, mientras que otros conciben su importancia en la facilidad para acceder a ofertas de trabajo y así lograr incrementar sus ingresos. Sin embargo, como mencionan Lugo, Kelly y Schurmann (2012), se deberá pensar en qué dispositivos pueden integrarse para obtener los mejores resultados no solo económicos, sino también en cuestiones de adaptabilidad y sostenibilidad. En este sentido, Fairlie y Bahr (2018) indican que algunos estudios encontraron que trabajadores con habilidades informáticas tenían salarios más altos.

Por otra parte, se distingue que el nivel académico tiene una influencia positiva en el ingreso personal mensual, pues un incremento de $10 \%$ en el nivel académico provocaría un incremento en el ingreso personal mensual de $2.4 \%$. Lo anterior concuerda con el análisis realizado por Preoţiuc, Volkova, Lampos, Bachrach y Aletras (2015) debido a que descubrieron correlaciones que muestran que un nivel educativo más alto indica mayores ingresos. Tal como mencionan Picatoste, Pérez y Ruesga (2018), el mayor nivel de educación en un país conlleva a un mayor nivel de ingreso per cápita, debido a que un mayor nivel de educación se traduce en una mayor productividad laboral y un mayor nivel de actuación en toda la sociedad. Por lo tanto, se considera, al igual que a diversos actores, al nivel educativo como una pieza fundamental para determinar el ingreso personal mensual. Diversos autores 


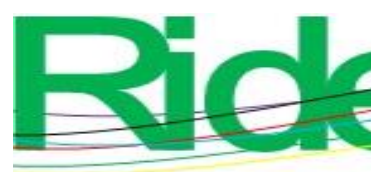

Revista Iberoamericana para la Investigación y el Desarrollo Educativo ISSN $2007-7467$

mencionan que al invertir en capital humano (educación y habilidades para el aprendizaje) se puede mejorar el ingreso y es considerada una de las formas más efectivas para reducir la desigualdad en el ingreso en el largo plazo (Jaumotte, Lall y Papageorgiou, 2008; Abdullah, Doucouliagos y Manning, 2011; Gruber y Kosack, 2013; Shahabadi et al., 2016). Además, se considera que un mayor nivel de educación permite un uso efectivo de la tecnología, lo que le otorga a su vez al individuo la posibilidad de acceder a diferentes sectores de la producción e incrementar su ingreso (Islam y Slack, 2016).

Por último, la edad es la variable que más influencia tiene en el ingreso mensual personal, pues un aumento de $10 \%$ en la edad, se traducirá en un incremento de $5 \%$ en el ingreso mensual personal. La edad tiene un papel importante en los ingresos, ya que se considera que, en promedio, a mayor edad mayor experiencia, capacitación o educación, lo que se traduce en mayores ingresos (Preoţiuc et al., 2015).

La presente investigación permite aclarar la importancia del nivel académico, la edad y el desarrollo de habilidades en relación con las TIC para que los individuos puedan incrementar sus ingresos. Sin embargo, en la muestra solo se contemplan los estudiantes universitarios que trabajan, por lo tanto, no se está contemplando a los trabajadores en general. La fortaleza principal de esta investigación es que puede constituir una base para poder realizar una caracterización de los trabajadores en México y las habilidades que poseen en las TIC y complementarlo con el diseño e implementación de programas educativos que permitan a los individuos acceder a mejores sus niveles de ingreso.

\section{Conclusiones}

Se encontró que el ingreso personal mensual de los estudiantes que trabajan se ve afectado negativamente por la propiedad de teléfono móvil y del uso de dispositivos móviles con fines escolares. Sin embargo, variables como la edad, los días de uso de las redes sociales y el nivel académico influyen positiva y significativamente en el ingreso económico. Esto concuerda con lo expuesto por otros autores debido a que el uso de las redes sociales es utilizado como un medio para complementar el ingreso; por otra parte, un nivel académico superior generalmente se traduce en una mayor productividad laboral y, por lo tanto, en un mayor nivel de ingreso. 


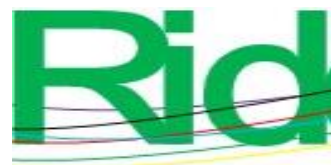

Los resultados de esta investigación permitieron identificar la importancia de mejorar el nivel de educación y las habilidades en las TIC de la población, principalmente las relacionadas con el uso de redes sociales, debido a que, para obtener los beneficios económicos y sociales de la adopción de estas herramientas, se deben diseñar, implementar y evaluar programas educativos apropiados que les permitan a los individuos desarrollar habilidades tecnológicas necesarias para el ambiente laboral.

Por lo anterior, el desarrollo de políticas encaminadas a un mejor acceso a las TIC, así como el desarrollo de capacidades para hacer uso de dichas tecnologías, permitirá a los individuos incrementar su ingreso económico, lo cual coadyuvará a la disminución de la desigualdad.

\section{Agradecimientos}

Instituto Politécnico Nacional, Proyecto SIP-20180645 "Estrategias digitales para aumentar el ingreso económico en comunidades con pobreza”. 


\section{Referencias}

Abdullah, A. J., Doucouliagos, H. and Manning, E. (2011). Education and Income Inequality: A Meta-Regression Analysis. (Unpublished manuscript). Deakin University.

Retrieved from https://pdfs.semanticscholar.org/3745/da57966971c0c86abae05c59eda3e28d170f.p df.

Álvarez, G., Vega, A. y Alvarez, G. (2011). Apropiación de las TIC en comunidades vulnerables: el caso de Medellín Digital. Apertura. Universidad de Guadalajara, $3(1)$.

Banco Mundial. (abril de 2014). Tecnologías de la Información y las Comunicaciones: Resultados del sector. Banco Mundial. Recuperado de http://www.bancomundial.org/es/results/2013/04/13/ict-results-profile.

Cacheiro, G. M. (2018). Educación y tecnología estrategias didácticas para la integración de las TIC. Madrid, España: UNED.

Dutton, W. and Reisdorf, B. (2016). Cultural divides and digital inequalities: Attitudes shaping internet and social media divides. Paper presented at the 44th Annual Telecommunication Policy Research Conference. Arlington, Virginia. Retrieved from https://papers.ssrn.com/sol3/papers.cfm?abstract_id=2756121.

Evangelista, R., Guerrieri, P. and Meliciani, V. (2014). The economic impact of digital technologies in Europe. Economics of Innovation and New Technology, 23(8), 802824.

Fairlie, R. and Bahr, P. (2018). The effects of computers and acquired skills on earnings, employment and college enrollment: Evidence from a field experiment and California IU earnings records. Economics of Education Review, 63, 51-63.

Frey, C. and Osborne, M. (September 2013). The future of employment: how susceptible are jobs to computerisation. Oxford Martin Program on Technology and Employment. Retrieved from https://www.oxfordmartin.ox.ac.uk/downloads/academic/future-ofemployment.pdf.

Gruber, L. and Kosack, S. (2013). The tertiary tilt: education and inequality in the developing world. World Development, 54, 253-272.

Gujarati, D. N. (2010). Econometría (5. a ed.). México: McGraw-Hill. 
Hernández, R. (2017). Impacto de las TIC en la educación: Retos y Perspectivas. Propósitos y representaciones, $15(1), 325-347$.

Hübler, M. and Hartje, R. (2016). Are smartphones smart for economic development? Economics Letters, 141, 130-133.

Huws, U., Spencer, N. and Syrdal, D. (2018). Online, on call: the spread of digitally organised just-in-time working and its implications for standard employment models. New Technology, Work and Employment, 33(2), 113-129.

Instituto Nacional de Estadística y Geografía [Inegi]. (2016). Gasto corriente monetario promedio trimestral por entidad federativa según grandes rubros del gasto, 2016. México: Instituto Nacional de Estadística y Geografía. Recuperado de https://www.inegi.org.mx/app/tabulados/pxweb/inicio.html?rxid=2a7423ff-cf364e8f-8cec-f04faba3b2a3\&db=Hogares\&px=Hogares_12.

Instituto Nacional de Estadística y Geografía [Inegi]. (2017). Disponibilidad y Uso de TIC. México: Instituto Nacional de Estadística y Geografía. Datos. Recuperado de https://www.inegi.org.mx/temas/ticshogares/.

Islam, M. and Slack, F. (2016). Women in rural Bangladesh: Empowered by access to mobile phones. Paper presented at the 9th International Conference on Theory and Practice of Electronic Governance. Montevideo, Uruguay.

Jaumotte, F., Lall, S. and Papageorgiou, C. (July 2008). Rising Income Inequality: Technology, or Trade and Financial Globalization? International Monetary Fund. Retrieved from https://www.imf.org/external/pubs/ft/wp/2008/wp08185.pdf.

Lugo, M. T., Kelly, V. y Schurmann, S. (2012). Políticas TIC en educación en América Latina: más allá del modelo 1:1. Campus Virtuales. Revista Científica de Tecnología Educativa, 1(01), 31-42.

Mendoça, S. and Crespo, N. (2015). Inequality in the network society: An integrated approach to ICT access, basic skills, and complex capabilities. Telecommunications policy, 39, 192-207.

Micheli, M. (2016). Social networking sites and low-income teenagers. Information, Communication \& Society, 19(5), 565-581.

Organización de las Naciones Unidas para la Educación, la Ciencia y la Cultura [Unesco]. (2015). Leveraging Information and Communication Technologies to Achieve the Post-2015 Education Goal. Report of the International Conference on ICT and Post- 
2015 Education. Qingdao, China: Organización de las Naciones Unidas para la Educación, la Ciencia y la Cultura.

Picatoste, J., Pérez, L. and Ruesga, S. M. (2018). A new educational pattern in response to new technologies and sustainable development. Enlightening ICT skills for youth employability in the European Union. Telematics and Informatics, 35, 1031-1038.

Preoţiuc, D., Volkova, S., Lampos, V., Bachrach, Y. and Aletras, N. (2015). Studying user income through language, behaviour and affect in social media. PLOS ONE, 10(9), 1-17. Retrieved from 0.1371/journal.pone.0138717.

Santos, M., Sequeira, T. and Ferreira, A. (2017). Income Inequality and Technological Adoption. Journal of Economic Issues, 51(4), 979-1000.

Serrano, C., Muñoz, J. and Brusca, I. (2018). A multivariate study of internet use and the digital divide. Social Science Quarterly, 99(4), 1409-1425.

Shahabadi, A., Nemati, M. and Hosseinidoust, S. E. (2016). The effect of knowledge economy factors on income inequality in the selected Islamic Countries. Journal of the knowledge economy, 8(4), 1174-1188.

Wu, L. (2013). Social network effects on productivity and job security: Evidence from the adoption of a social networking tool. Information Systems Research, 24(1), 30-51.

Yeaple, S. (2005). A Simple Model of Firm Heterogeneity, International Trade, and Wages. Journal of International Economics, 65, 1-20. 


\begin{tabular}{|c|c|}
\hline Rol de Contribución & Autor (es) \\
\hline Conceptualización & Martha Jiménez García y María Vianey Espejel García (igual) \\
\hline Metodología & María Vianey Espejel García y Martha Jiménez García (igual) \\
\hline Software & María Vianey Espejel García \\
\hline Validación & Martha Jiménez García \\
\hline Análisis Formal & María Vianey Espejel García \\
\hline Investigación & Martha Jiménez García \\
\hline Recursos & Martha Jiménez García \\
\hline Curación de datos & María Vianey Espejel García y Martha Jiménez García (igual) \\
\hline $\begin{array}{l}\text { Escritura - Preparación del borrador } \\
\text { original }\end{array}$ & María Vianey Espejel García (principal), Martha Jiménez García (apoya) \\
\hline Escritura - Revisión y edición & María Vianey Espejel García \\
\hline Visualización & María Vianey Espejel García \\
\hline Supervisión & Martha Jiménez García \\
\hline Administración de Proyectos & Martha Jiménez García y María Vianey Espejel García (igual) \\
\hline Adquisición de fondos & Martha Jiménez García \\
\hline
\end{tabular}

